\title{
PRÁCTICAS CULINARIAS ASOCIADAS AL CONSUMO DE FRIJOLES EN FAMILIAS COSTARRICENSES ${ }^{1}$
}

\author{
Shirley Rodríguez-González², Xinia Elena Fernández-Rojas ${ }^{2}$
}

\begin{abstract}
RESUMEN
Prácticas culinarias asociadas al consumo de frijoles en familias costarricenses. El objetivo de este trabajo fue identificar las prácticas asociadas al consumo de frijoles en una población rural y una urbana de Costa Rica. El estudio fue desarrollado en el 2005, en una muestra de 475 familias que acostumbran preparar y consumir frijoles (223 de la zona rural de Jesús de Atenas y 252 de la zona urbana en La Unión de Tres Ríos). En estas familias se entrevistó a la persona encargada de la preparación de los alimentos. Este estudio mostró un consumo per cápita de frijoles de 37,5 g/p/ día de peso crudo. El consumo per cápita por área geográfica, fue mayor en zona rural $(46,8+25 \mathrm{~g} / \mathrm{p} / \mathrm{d})$ que en la zona urbana $(37,5+23 \mathrm{~g} / \mathrm{p} / \mathrm{d})(\mathrm{F}=18,031, \mathrm{p}=0,000)$. Los factores asociados al consumo se diferenciaron por zona; en la rural se relacionaron con algunas características de la persona que prepara los alimentos, prácticas de preparación de los frijoles y con la razón de consumo manifestada, mientras que en la zona urbana los factores asociados estuvieron relacionados únicamente con las prácticas culinarias.
\end{abstract}

Palabras claves: hábitos alimentarios, área geográfica, zona rural, zona urbana.

\begin{abstract}
Culinary practices associated with bean consumption in Costa Rican families. The aim of this study was to identify culinary practices associated with bean consumption in families from one rural population (Jesus of Atenas) and one urban (La Union of Tres Rios) of Costa Rica. The study was developed in 2005, in a sample of 475 families that usually prepare and eat beans (223 rural and 252 urban). In this families, the person responsible for the preparation of food was interviewed. The study showed a per capita beans consumption of 37.5 $\mathrm{g} / \mathrm{person} /$ day $(\mathrm{g} / \mathrm{p} / \mathrm{d})$ of raw weight. This consumption differs by geographic condition, being higher in rural area $(46.8+25 \mathrm{~g} / \mathrm{p} / \mathrm{d})$ than in urban areas $(37.5+23 \mathrm{~g} / \mathrm{p} / \mathrm{d})(\mathrm{F}=$ $18.031, \mathrm{p}=0.000)$. The factors that were associated with consumption are different by area; in the rural area the factors were some characteristics of the person preparing bean, some preparation practices and the reasons manifested for consumption, whereas in urban areas the associated factors were only related to preparation practices.
\end{abstract}

Keywords: food habits, geographical area, rural, urban.

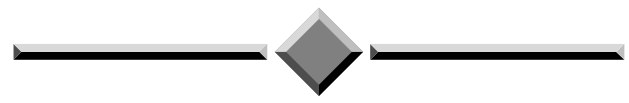

\footnotetext{
1 Recibido:13 de marzo, 2014. Aceptado: 22 de julio, 2014. Parte de la tesis para optar por el grado de Magíster Scientiae en Nutrición Humana de la primer autora, Universidad de Costa Rica, dentro del proyecto "Estudio de las características físico-químicas y nutricionales de frijoles (Phaseolus vulgaris)".

2 Universidad de Costa Rica, Escuela de Nutrición. Dirección Postal: 2060, Ciudad Universitaria Rodrigo Facio, San Pedro, Montes de Oca, Costa Rica. shirley.rodriguez@ucr.ac.cr, xinia.fernandezrojas@ucr.ac.cr
}

(C) 2015 Agronomía Mesoamericana es desarrollada en la Universidad de Costa Rica y se encuentra licenciada con Creative Commons Reconocimiento-NoComercial-SinObraDerivada 3.0 Costa Rica. Para más información escríbanos a pccmca@ucr.ac.cr 


\section{INTRODUCCIÓN}

El frijol común (Phaseolus vulgaris) es una de las fuentes más importantes de energía y de nutrientes a nivel mundial, especialmente en países en vías de desarrollo (Rodríguez y Fernández-Rojas, 2003; Serrano y Goni, 2004). Los frijoles son fuente de proteína, vitaminas (tiamina y ácido fólico), minerales (potasio, magnesio, zinc, hierro y fósforo) y además, de carbohidratos y fibra (Messina, 1999; Maldonado y Sammán, 2000; Menchú y Méndez, 2006; Ramírez y Rangel, 2011). Según Guzmán et al. (1996), Andlauer et al. (1998), Rodríguez y Fernández-Rojas (2003), y Ramírez y Rangel (2011) los frijoles aportan fitoquímicos, los cuales se han identificado como importantes para la salud, ya que protegen contra algunos de los factores que estimulan el crecimiento de tumores y el envejecimiento de los tejidos, y reducen el riesgo de padecer otras enfermedades crónico-degenerativas.

En Costa Rica, el frijol es consumido por el 97\% de los costarricenses (Ministerio de Salud, 1996), sin embargo, la cantidad que se consume ha disminuido en los últimos años (Rodríguez y Fernández-Rojas, 2003).

A pesar de que el frijol es un alimento básico en la dieta costarricense, hay muy poca información sobre los factores que condicionan, promueven o inhiben su consumo.

Los únicos trabajos documentados fueron realizados por Campos et al. (1999) y Martínez et al (2000), el cual se circunscribió a una población urbana de clase media, por lo que el estudio de prácticas relacionadas con el manejo de frijoles en el hogar, permitiría tener conocimiento de las prácticas que son realizadas por dicha población, lo que podría ser un insumo importante a ser considerado para establecer recomendaciones en programas de educación nutricional que permitan preservar el mayor contenido de nutrientes y aumentar el consumo de frijoles. Una mejor preparación y un mayor consumo de este alimento mejorará el aporte de nutrientes a la dieta del costarricense, lo que se traducirá en un beneficio para la salud de la población.

El objetivo de este trabajo fue identificar las prácticas asociadas al consumo de frijoles en una población rural y una población urbana de Costa Rica.

\section{MATERIALES Y MÉTODOS}

El estudio se desarrolló en el 2005 en una población rural y una población urbana de Costa Rica. Este fue un estudio de tipo transversal y descriptivo con enfoque cuantitativo que buscaba determinar las prácticas culinarias que están asociadas con el consumo de frijoles en dichas zonas y que podrían afectar la calidad nutricional del grano.

La población estuvo constituida por las familias de las comunidades de Jesús de Atenas, población rural y de La Unión de Tres Ríos, población urbana. La muestra fue seleccionada a partir de un muestreo estratificado por conglomerados según condición geográfica. Se obtuvo un total 475 familias, 223 en la zona rural y 252 en la zona urbana. En dichas familias se entrevistó la persona responsable de preparar y de conservar los alimentos en el hogar y que voluntariamente aceptó participar en el estudio. Se partió del supuesto de que la persona que prepara los alimentos en el hogar también prepara los frijoles para el consumo de la familia. Entre las limitaciones del estudio pueden citarse: no se midió el consumo de frijoles de cada miembro de la familia, sólo se estimó, tampoco el consumo de frijoles fuera del hogar y no se determinaron los conocimientos y percepciones con respecto a los frijoles de la persona encargada de preparar los alimentos.

Las variables estudiadas fueron:

1. Localización geográfica.

2. Ocupación del jefe de hogar.

3. Característica de la persona encargada de preparar los alimentos en el hogar.

4. Prácticas de consumo de frijoles: consumo de frijoles, frecuencia de consumo, frecuencia diaria, tiempos de comida, calidad del grano y color del grano.

5. Razones de consumo o no consumo.

Estas variables fueron definidas a partir del referencial teórico y de estudios precedentes sobre prácticas asociadas a la preparación, consumo y almacenamiento de frijoles en Costa Rica.

Para recolectar los datos se elaboró un cuestionario semiestructurado que fue validado en una población similar y que posteriormente fue aplicado a la muestra. Considerando las variables del estudio, este cuestionario permitió obtener la información referente a las características generales de la familia, 
de la persona encargada de preparar los alimentos y las prácticas de consumo de frijoles en las familias.

Todas las variables en estudio fueron analizadas a partir de estadística descriptiva e inferencial con el fin de establecer asociación con el consumo promedio por persona por día, determinada como variable dependiente del estudio.

Para establecer el consumo promedio por persona, se determinó la cantidad cocida la última vez, y se dividió entre el número de días de su consumo $\mathrm{y}$ posteriormente entre el número de personas que consumen frijoles en el hogar mayores a seis meses.

Para determinar los factores asociados al consumo, se aplicó una regresión lineal múltiple a partir de las variables que mostraron correlación con el consumo, considerada variable dependiente. Los datos fueron analizados con el programa estadístico SPSS versión 12.

\section{RESULTADOS Y DISCUSIÓN}

Esta investigación permitió afirmar lo mostrado en otros estudios (Abarca et al., 1996; Robertson, 1996; Morúa y Villalobos, 1997; Campos et al., 1999), al identificar el frijol como un alimento básico en la población costarricense.

El estudio reveló un consumo de frijoles de $37,5 \mathrm{~g}$ en crudo por persona por día (g/p/día). Este consumo difiere estadísticamente $(\mathrm{F}=18,03, \mathrm{p}=0,000)$ por condición geográfica, en la zona rural fue de 46,8 \pm 25 gramos, mientras que en la zona urbana fue de $37,5 \pm 23$ gramos. Estos resultados son similares a los encontrados en la última Encuesta Nacional de Consumo de Alimentos donde se presentó el mismo comportamiento por zona. Sin embargo, al comparar los datos puede observarse un mayor consumo en las zonas incluidas en este estudio, pues en la Encuesta Nacional de Consumo de Alimentos se determinó un promedio por persona por día (peso crudo) de 38,6 $\mathrm{g}, 32 \mathrm{~g}$ para la zona urbana y $43 \mathrm{~g}$ para la zona rural (Ministerio de Salud, 2001).

Es importante rescatar que este resultado puede obedecer a diferencias en la forma de cálculo del consumo, pues en las encuestas realizadas a nivel nacional se reporta una estimación del consumo de frijoles en la semana anterior, mientras que en este estudio se reporta en el tiempo transcurrido desde el momento en que se cocinaron los frijoles y el momento que se acabaron los mismos.
La frecuencia semanal de consumo fue de 6,3 $( \pm$ $1,51)$, siendo mayor la frecuencia presentada en la zona rural $(6,5 \pm 1,26)$ que la presentada en la zona urbana $(6,2 \pm 1,70)$. La diferencia presentada entre zonas es estadísticamente significativa $(\mathrm{F}=4,932, \mathrm{p}=0,027)$.

La mayoría de las familias consumió los frijoles diariamente, lo que coincidió con resultados reportados en varias investigaciones realizadas en nuestro país (Chinnock, 1992; Robertson, 1996; Martínez et al. 2000; Dumani y Páez, 2004). Esta práctica de consumo se consideró como ideal, de acuerdo con lo recomendado por Martínez et al. (2000) en un estudio realizado con población urbana.

En la zona rural, la diferencia entre el consumo y la frecuencia fue significativa, pero no fue así para la zona urbana. Dato que difiere con el presentado por Dumani y Páez (2004) quienes establecieron una relación directa entre la cantidad consumida de frijoles y la frecuencia semanal de consumo en una investigación realizada en una población urbana.

Al comparar el consumo de frijoles con características como la edad de la persona que prepara los alimentos, se encontró que la cantidad consumida de frijoles fue mayor en la zona rural para las tres clasificaciones de edad realizadas (Figura 1). En ambas zonas, la cantidad consumida de frijoles es mayor cuando la persona que prepara los frijoles es de mayor edad (Figura 1). Sin embargo, al realizar el análisis de correlación entre el consumo y la edad en la zona rural, se encontró una correlación directa entre ambas variables $(\mathrm{r}=0,212, \mathrm{p}=0,001)$, no así para la zona urbana $(\mathrm{r}=0,099, \mathrm{p}=0,118)$.

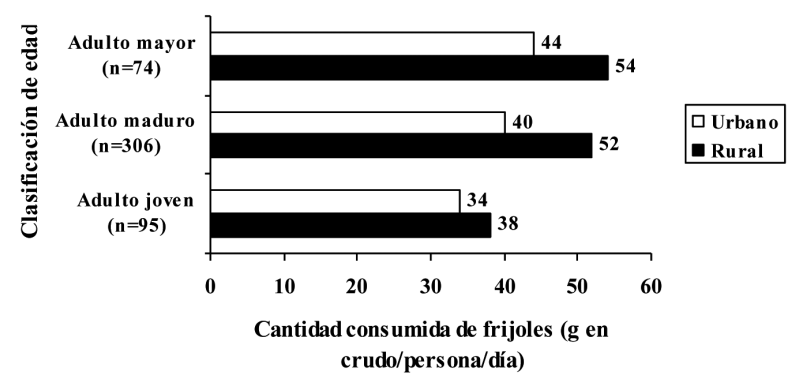

Figura 1. Cantidad consumida de frijoles según edad de la persona encargada de preparar los alimentos en el hogar, en una población urbana y otra rural. Jesús de Atenas y La Unión de Tres Ríos, Costa Rica. 2005. 
Lo anterior contrasta con lo reportado en varios estudios, que señalan que los niños tienden a imitar el comportamiento alimentario de los adultos con los que conviven (Olvera y Givaudan, 1984; Fischer, 1989; Plazas, 1995; Domínguez-Vásquez et al., 2008). Birch y Gimm-Thomas (1997) refuerzan esta aseveración, al afirmar que la forma en que los padres manejan su propio consumo de alimentos tiene un gran impacto sobre la aceptación, las preferencias y los patrones alimentarios de los hijos.

En la zona rural, la cantidad consumida de frijoles aumenta conforme disminuye el nivel educativo de la persona encargada de preparar los alimentos, rompiéndose esta tendencia en las personas que presentan un nivel educativo universitario incompleto. Igualmente en la zona urbana, el mayor consumo se observó en el grupo con primaria incompleta; aunque igualmente el consumo aumentó en el grupo de mayor nivel educativo (Figura 2).

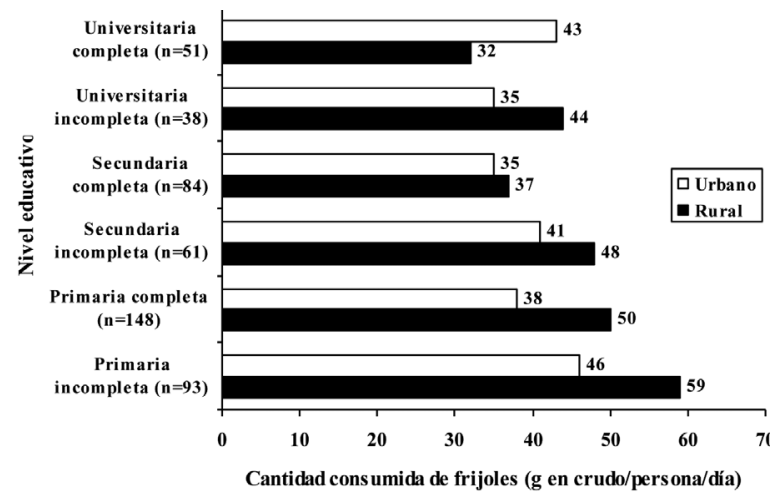

Figura 2. Cantidad consumida de frijoles según nivel educativo de la persona encargada de preparar los alimentos en el hogar, en una población urbana y otra rural. Jesús de Atenas y La Unión de Tres Ríos, Costa Rica. 2005.

Esta situación es esperada, debido a que las personas con menor nivel educativo, por lo general, trabajan únicamente en el hogar y puede destinar más tiempo para realizar la preparación de los alimentos. Según Contreras (1993), Garcia (1997), Rodrigues y Sabes (2006) y Schlindwein y Kassouf (2007) cuando la mujer, quien es en la mayoría de las veces la responsable por la alimentación de la familia, se incorpora al mercado laboral, dispone de menos tiempo para realizar la preparación de los alimentos, recurre a alimentos de fácil preparación o por la alimentación fuera de casa. Además, el trabajo fuera del hogar dificulta la realización de tiempos de comida de la familia completa y la supervisión de los alimentos que consumen los niños (Campos et al., 1999).

En las familias de la zona urbana, la cantidad consumida promedio de frijoles en las diversas categorías del nivel educativo, es menor que el presentado en la zona rural, excepto para el nivel universitario completo, donde la cantidad consumida de frijoles en las familias de la zona urbana presentó una diferencia de $10,7 \mathrm{~g}$ con respecto al consumo en esa misma categoría en la zona rural.

Los resultados reflejan una correlación inversa entre el nivel de escolaridad y la cantidad consumida promedio de frijoles $(\mathrm{r}=-0,290, \mathrm{p}=0,000)$ en la zona rural (a mayor nivel educativo menor es el consumo de frijoles). Resultado coincidente con los datos encontrados por Schlindwein y Kassouf (2007) y Coelho et al. (2009). No obstante, en la zona urbana se mostró una ausencia de correlación entre esas variables $(\mathrm{r}=-0,045, \mathrm{p}=0,475)$.

Este comportamiento en la zona urbana, podría deberse al hecho de que tener un mayor nivel educativo facilita informarse sobre los beneficios de este alimento (Wolfe y Campbell, 1993; Manço y Costa, 2004); además, existe una mayor preocupación por la salud. Según Campos et al. (1999), las madres de la zona urbana estudiada conocen sobre el valor nutritivo de los frijoles, lo cual favorece su consumo.

\section{Contribución relativa de los factores asociados al consumo}

Los factores que están asociados al consumo de frijoles en la población estudiada, están relacionados con la condición geográfica (rural o urbana), la frecuencia de cocción de los frijoles por mes y la cantidad cocinada de frijoles por ocasión (Cuadro 1).

Del modelo se tiene que si la zona de residencia es la rural, el consumo aumenta en $10,887 \mathrm{~g}$ en comparación con la zona urbana, manteniendo constantes las otras variables, y que por cada gramo de incremento en la cantidad cocinada de frijoles, aumenta el consumo en $0,026 \mathrm{~g}$, manteniendo constante todas las otras variables. 
Cuadro 1. Modelo propuesto para estudiar el comportamiento del consumo de frijoles en las poblaciones de Jesús de Atenas (rural) y La Unión de Tres Ríos (urbana), Costa Rica. 2005.

\begin{tabular}{lccc}
\hline Modelo & $\begin{array}{c}\text { Coefi- } \\
\text { ciente B }\end{array}$ & t & P \\
\hline $\begin{array}{l}\text { Frecuencia de cocción de } \\
\text { frijoles por mes }\end{array}$ & 1,424 & 4,605 & 0,000 \\
$\begin{array}{l}\text { Cantidad cocinada } \\
\text { de frijoles por ocasión }\end{array}$ & 0,026 & 3,346 & 0,000 \\
Zona de residencia & 10,887 & 4,053 & 0,001 \\
\hline
\end{tabular}

$\mathrm{R}=0,458, \mathrm{R}^{2}$ Ajustado $=0,205(\mathrm{P}=0,000)$.

Con respecto a la frecuencia de cocción de frijoles por mes se tiene que por cada vez que se incremente la frecuencia, se produce un aumento de $1,424 \mathrm{~g}$ en el consumo de frijoles, si se mantienen todas las otras variables constantes.

Considerando la importancia de la zona de residencia se analizaron los factores para cada una de ellas y las variables que determinan el consumo difieren por zona (Cuadro 2). En la zona rural se incluyeron seis variables en el modelo, el cual explica el 36\% de la variabilidad del consumo, mientras que en la zona urbana, se incluyeron tres variables y este explica el $20 \%$ el consumo de frijoles.

Los resultados muestran que en la zona rural los factores que estuvieron asociados al consumo, se relacionaron con algunas características de la persona que prepara los alimentos y a prácticas asociadas con la preparación, tales como la frecuencia de cocción por mes, la cantidad cocinada por ocasión, el tiempo de cocción y de remojo. En la zona urbana, los factores asociados al consumo de frijoles estuvieron relacionados únicamente con la preparación del mismo (frecuencia de cocción, y cantidad de cocción y tiempo de remojo). Las variables que más contribuyeron a explicar el consumo de frijoles en la población rural, fueron la escolaridad de la persona que prepara los alimentos, el tiempo de remojo y la cantidad cocinada por ocasión.

En la zona rural la regresión mostró que, por cada año más de edad en la persona encargada de preparar los alimentos en el hogar, disminuyó en 0,362 g el consumo de frijoles; en relación con la escolaridad,
Cuadro 2. Modelo propuesto para estudiar el comportamiento del consumo de frijoles según condición geográfica. Jesús de Atenas (rural) y La Unión de Tres Ríos (urbana), Costa Rica. 2005.

\begin{tabular}{lccc}
\hline Modelo & $\begin{array}{c}\text { Coefi- } \\
\text { ciente B }\end{array}$ & t & P \\
\hline Zona rural* & & & \\
$\begin{array}{l}\text { Edad de la persona que prepara } \\
\text { alimentos }\end{array}$ & $-0,362$ & $-1,854$ & 0,069 \\
Escolaridad de la persona que & & & \\
prepara alimentos & $-2,712$ & $-3,443$ & 0,001 \\
Frecuencia de cocción por mes & 1,824 & 2,012 & 0,049 \\
Cantidad cocinada por ocasión & 0,023 & 2,499 & 0,016 \\
Tiempo de cocción & 0,039 & 1,976 & 0,053 \\
Tiempo de remojo & $-1,267$ & $-2,589$ & 0,012 \\
Zona urbana** & & & \\
Frecuencia de cocción por mes & 1,366 & 3,819 & 0,000 \\
Cantidad cocinada por ocasión & 0,032 & 4,695 & 0,000 \\
Tiempo de remojo & 0,762 & 1,988 & 0,049 \\
\hline & & & \\
\hline
\end{tabular}

$* \mathrm{R}=0,583, \mathrm{R}^{2}$ Ajustado $=0,365(\mathrm{p}=0,001)$.

$* * \mathrm{R}=0,453, \mathrm{R}^{2}$ Ajustado $=0,197(\mathrm{p}=0,000)$.

por cada año más de estudios disminuyó en 2,712 g el consumo de frijoles. Así mismo, cuando la frecuencia de cocción por mes aumentó en una vez, el consumo de frijoles se incrementó en 1,824 g. Por su parte, al aumentar en $1 \mathrm{~g}$ la cantidad de frijoles cocinada por ocasión, el consumo de frijoles aumentó en un 0,023 g; de la misma forma, conforme el tiempo de cocción se incrementó en un minuto el consumo también se incrementó en $0,039 \mathrm{~g}$. Por último, el consumo de frijoles disminuyó en 1,267 g al incrementar el tiempo de remojo en una hora.

En la zona urbana la regresión indicó que, al mantener todas las variables constantes excepto la estudiada, el consumo de frijoles se incrementó en 1,366 g al aumentar en una vez la frecuencia de cocción por mes; si la cantidad cocinada por ocasión aumenta en $1 \mathrm{~g}$, el consumo de frijoles se incrementa en 0,032 g. Por su parte, si el tiempo de remojo aumenta en una hora el consumo de frijoles se incrementa en 0,762 g.

Este estudio permite concluir que los factores que estuvieron asociados al consumo, se diferenciaron por zona, en la rural los factores que estuvieron 
asociados se relacionaron con algunas características de la persona que prepara los alimentos, con algunas prácticas de preparación de los frijoles y con la razón de consumo manifestada, mientras que en la zona urbana los factores asociados estuvieron relacionados únicamente con prácticas de preparación, los cuales deben ser considerados cuando se requieren realizar intervenciones para promover el consumo de frijoles. Dicha información puede brindar aportes potenciales para la elaboración de programas que promuevan el consumo de este alimento a nivel nacional.

En relación a las estrategias de promoción, considerando los resultados del estudio, estas deberían considerar las diferencias encontradas. Así, en ambas zonas, es recomendable exaltar la importancia de este alimento en la salud de las personas. Pero en el caso de la zona rural es necesario abordar a las poblaciones jóvenes y de clase media y alta, debido a que hay una asociación importante entre ellas y un menor consumo de frijoles.

\section{LITERATURA CITADA}

Abarca, L., D. Chaves, M. Dumani, F. Elizondo, R. Flores, y R. Riggioni. 1996. Prácticas alimentarias de seis grupos de mujeres de la Gran Área Metropolitana. Seminario Lic, Universidad de Costa Rica, San José, Costa Rica.

Andlauer, A., C. Wilfried, V. Stehle, P. Peter, G. Furst, y D. Peter. 1998. Chemoprevention - a novel approach in diabetics. Clin. Nut. Met. Car. 1(6):539-547.

Birch, L., y K. Grimm-Thomas. 1997. Patrones de aceptación de los alimentos: los niños aprenden lo que viven. Pediatr. Basic. 18:2-9.

Campos, E., A. Fernández, I. Sánchez, T. Sancho, y C. Villalobos. 1999. Diagnóstico para el diseño de una campaña educativa tendiente a incrementar el consumo de frijol en una comunidad urbana de clase media de Costa Rica. Seminario Lic, Universidad de Costa Rica, San José, Costa Rica.

Coelho, A., D. Aguiar, y E.A. Fernandes. 2009. Padrão de consumo de alimentos no Brasil. Rev. Econ.Soc. Rural 47:335-362.

Contreras, J. 1993. Antropología de la alimentación. Editorial Eudema, Madrid, España.

Chinnock, A. 1992. Tamaño de la porción de algunos alimentos y preparaciones para preescolares y adultos de un área rural y una comunidad urbana del Valle Central de Costa Rica. Instituto de Investigaciones en Salud, Universidad de Costa Rica, San José, Costa Rica.

Domínguez-Vásquez, P., S. Olivares, y J.L. Santos. 2008. Influencia familiar sobre la conducta alimentaria y su relación con la obesidad infantil. ALAN 58(3):249-255.

Dumani, M., y P. Páez. 2004. Efectos de una campaña educativa sobre frijoles en un grupo de mujeres. Agron. Mesoam. 15:379-392.

Fisher, M. 1989. La alimentación del preescolar: formación de hábitos alimentarios. Avances en Supervivencia Educativa 7(3):3.

Garcia, R.W.D. 1997. Práticas e comportamento alimentar no meio urbano: um estudo no centro da cidade de São Paulo. Cad. Saúde Pública 13:455-467.

Guzmán, M., H. Salvador, L. Zaragoza, y M. Juárez. 1996. Remojo de dos sales y tiempo de cocción de tres variedades de frijol. Agrociencia 30:201-205.

Maldonado, S., y N. Sammán. 2000. Composición química y contenido de minerales de leguminosas y cereales producidos en el noreste argentino. ALAN 50(2):1-10.

Manço, A.N., y Costa, F.N. 2004. Educação nutricional: caminhos possíveis. Alim. Nutr., Araraquara 15(2):145-153.

Martínez, T., A. Murillo, S. Rodríguez, y P. Páez. 2000. Elaboración de los contenidos de los mensajes de una Campaña Educativa para aumentar el consumo de frijoles en una comunidad urbana de clase media. Sem. Lic, Universidad de Costa Rica, San José, Costa Rica.

Menchú, M.T., y H. Méndez. 2006. Tabla de composición de alimentos de Centroamérica. 2 ed. INCAP/OPS, Guatemala.

Messina, M. 1999. Legumes and soybeans: Overview of their nutritional prolifes and health effects. Am. J. Clin. Nut. (Suppl). 70:439S-450S.

Ministerio de Salud. 1996. Encuesta Nacional de Nutrición: Fascículo 3 Consumo Aparente. Ministerio de Salud, San José, Costa Rica.

Ministerio de Salud. 2001. Encuesta Nacional de Consumo de Alimentos. Ministerio de Salud, San José, Costa Rica.

Morúa, H., y K. Villalobos. 1997. Disponibilidad, acceso a los alimentos, conocimientos, actitudes y prácticas alimentarias de las familias de la comunidad de Lousiana, Cairo, Siquirres. Tesis Lic, Universidad de Costa Rica, San José, Costa Rica.

Olvera, M., y M. Givaudan. 1984. La formación de hábitos alimentarios en la infancia. Cuadernos de Nutrición 7(5):39-43. 
Plazas, M. 1995. Que los niños aprendan y disfruten de una buena alimentación. Cuadernos de Nutrición 18(2): 17-19.

Ramírez, J.C.R., y I.B.E.U. Rangel. 2011.El frijol (Phaseolus vulgaris): su importancia nutricional y como fuente de fitoquímicos. Revista Fuente 3(8):5-9.

Robertson, A. 1996. Conocimientos, actitudes y prácticas de madres en relación con alimentos fuente de hierro en la dieta de los niños menores de tres años en un área rural y urbana de la provincia de San José. Tesis Lic, Universidad de Costa Rica. San José, Costa Rica.

Rodríguez, L., y X.E. Fernández-Rojas. 2003. Los frijoles (Phaseolus vulgaris): su aporte a la dieta del costarricense. Acta Médica Costarricense 45(3):120-125.
Rodrigues, F.S., y J.J.S. Sabes. 2006. A percepção do consumidor de alimentos "fora de casa": um estudo multicaso na cidade de Campo Grande/MS. Caderno de Administração 14(2):37-45.

Schlindwein, M.M., y A.L. Kassouf. 2007. Influência do custo de oportunidade do tempo da Mulher sobre o padrão de consumo alimentar no Brasil. Pesquisa e Planejamento Econômico 37:489-520.

Serrano, J., y I. Goni. 2004. Papel del frijol negro Phaseolus vulgaris en el estado nutricional de la población guatemalteca. ALAN 54(1):36-44.

Wolfe, W., y C. Campbell. 1993. Food pattern, diet quality and related characteristics of schoolchildren in New York State. J. Am. Diet. Assoc. 93:1280-1284. 
